\title{
Regeneración ósea fisiológica en ameloblastoma mandibular con manejo conservador, seguimiento de 8 años
}

\author{
DOI: $10.5377 /$ alerta.v3i2.9631
}

Jorge Eugenio Flores Valdez ${ }^{1}$, Amílcar Antonio Barillas Torres ${ }^{2}$, Katya Michelle Barillas Prieto ${ }^{3}$, Wendy Escobar De González ${ }^{4}$, Ruth Fernández de Quezada ${ }^{5}$

1. Hospital Nacional Rosales, Facultad de Odontología Universidad de El Salvador, San Salvador, El Salvador.

2. Hospital Nacional Rosales San Salvador, El Salvador.

3. Facultad de Odontología, Universidad Evangélica de El Salvador, San Salvador, El Salvador.

4,5. Centro de Investigaciones de la Facultad de Odontología, Universidad de El Salvador, San Salvador, El Salvador.

${ }^{*}$ Correspondencia

Meugenio.flores@ues.edu.sy

a

Physiological bone regeneration from Maxillary Ameloblastoma with conservative intervention. Follow up for 8 years.

\section{Citación recomendada:}

Flores-Valdez JE, Barillas-Torres AA, Barillas-Prieto KM, Escobar de González W, Fernández de Quezada R. Regeneración ósea fisiológica en ameloblastoma mandibular con manejo conservador, seguimiento de 8 años. Alerta. 2020; 3(2):50-56 DOI: https://doi.org/10.5377/ alerta.v3i2.9631

\section{Recibido:}

16 de abril de 2020

\section{Aceptado:}

9 de julio de 2020

\section{Publicado:}

17 de julio de 2020

\section{Contribución de autoría:}

JEFV': Búsqueda bibliográfica, redacción y revisión

final de artículo. AABT2: procedimientos a paciente, controles postquirúrgicos hospitalarios $\mathrm{KMBP}^{3}$. Ortodoncia, controles y rehabilitación protésica WEdG ${ }^{4}$ $\mathrm{RFdQ}^{5}$ : Redacción y revisión de caso clínico.

\section{Conflicto de intereses:}

Los autores declaran no tener conflicto de interés

\footnotetext{
1. (D) 0000-0003-0911-8956

2. (D) 0000-0002-9252-1465

3. (1) 0000-0001-8085-3203

4. (1) 0000-0001-9725-0783

5. (1) 0000-0002-2869-584X
}

\section{Resumen}

El ameloblastoma es un tumor de origen odontogénico, con un comportamiento altamente agresivo. Estos pueden ser sólidos o multiquístico, uniquístico, periférico o extraóseo y desmoplásico. Dentro de las alternativas de manejo, se prefiere un tratamiento que garantice seguridad para el paciente, como la resección radical o en bloque. Cada vez se utiliza menos el tratamiento conservador debido a una alta tasa de recidiva. Se presenta un caso de paciente masculino de 13 años, que fue visto en el Hospital Nacional Rosales, en el servicio de cirugía maxilofacial de El Salvador, por una inflamación en la región posterior mandibular en el lado izquierdo de la cara. El examen intraoral reveló leve extrusión y movilidad grado 2 de pieza 36 y 37. Los análisis clínicos, radiográficos e histopatológicos confirmaron el diagnóstico de ameloblastoma. Fue tratado de forma conservadora mediante curetaje, ostectomía periférica y marsupialización. Se tuvo buen resultado estético y funcional, con un adecuado proceso de cicatrización y regeneración ósea fisiológica, sin la necesidad de materiales de injerto óseo. Después de un seguimiento de 8 años, no hubo evidencia de recurrencia. Esta decisión depende de la variante clínica, la extensión tumoral y el adecuado control postoperatorio del paciente.

\section{Palabras clave}

Ameloblastoma, tumores odontogénicos, cirugía maxilofacial, El Salvador.

\begin{abstract}
Ameloblastoma is a tumor of odontogenic origin and highly aggressive. Four clinical variants of ameloblastomas are known: solid or multicystic, unicystic, peripheral or extra- osseous and desmoplastic. Among the treatment alternatives, a treatment that ensures a high percentage of successful prognosis for the patient is preferred, being the most indicated, radical resection, or in bulk. Conservative treatment is less used due to its recurrence rate. A clinical case of a 13-year-old male patient is presented to the Rosales National Hospital, in the Maxillofacial Surgery service of El Salvador with an inflammation in the posterior mandibular region on the left side of the face. Intraoral examination revealed mild extrusion and mobility grade 2 of parts 37 and 36. Clinical, radiographic and histopathological analyzes confirmed the diagnosis of Ameloblastoma. It was treated conservatively by curettage, peripheral osteotomy and marsupialization. A good aesthetic and functional result was obtained, applying the conservative technique, with an adequate healing and physiological bone regeneration, without recourse to bone grafting materials. After an 8-year follow-up no evidence was found of tumor recurrence. The decision regarding the type of treatment to be applied, depends on the clinical variables, tumor extension and adequate postoperative monitoring and care of the patient.
\end{abstract}

Keywords

Ameloblastoma, Odontogenic tumors, Maxillofacial surgery, El Salvador 


\section{Introducción}

El ameloblastoma es un tumor odontogénico epitelial benigno de crecimiento lento, localmente agresivo. Se origina en los restos epiteliales de la lámina dental, el órgano del esmalte, las células basales de la mucosa oral o el revestimiento epitelial de un quiste odontogénico ${ }^{1,2}$. En cuanto a su prevalencia, constituye el $1 \%$ de todos los tumores o quistes maxilares y el $11 \%$ de los tumores odontogénicos mandibulares ${ }^{3,4}$. En su comportamiento clínico se pueden clasificar cuatro tipos de ameloblastomas: ameloblastoma intraóseo, sólido o multiquístico; ameloblastoma uniquistico; ameloblastoma periférico o extraóseo; y ameloblastoma desmoplásico 2,5 . Los ameloblastomas malignos son extremadamente raros; en la clasificación de la Organización Mundial de la Salud (OMS) se reconocen dos subtipos principales: el ameloblastoma metastásico y el carcinoma ameloblástico ${ }^{6,7}$.

Un meta-análisis acerca de la incidencia global y el perfil epidemiológico del ameloblastoma en el 2019 mostró una tasa de incidencia de 0.92 por 1000000 personas al año ${ }^{8}$, superior al 0.5 que presentó la OMS en su clasificación de tumores de cabeza y cuello del 20172,6.

El Ameloblastoma tiende más predilección en hombres que en mujeres, afecta más en la tercera década de vida ${ }^{1,8,9}$ y tiende a afectar más la región posterior de mandíbula que el maxilar $2,8,9$. En el $40 \%$ de los casos hay asociación de un diente no erupcionado, usualmente el tercer molar inferior ${ }^{6}$.

En un estudio, se menciona al ameloblastoma de tipo sólido multiquístico como el más común; de 2462 casos representó el $67.7 \%$. Los tipos uniquísticos, desmoplásicos y periféricos representaron el $26.2 \%$ (953 casos), $3.6 \%$ (130) y $1.0 \%$ (38) de todos los casos, respectivamente ${ }^{8}$.

Clínicamente, el tumor crece de un modo lento y silente, sin originar signos ni síntomas en sus estadios más precoces. En estadios de evolución avanzados puede provocar migración, desviación y movilidad de piezas dentarias, como también reabsorción radicular y parestesia. Puede expandir corticales, aunque habitualmente las erosiona invadiendo el tejido blando 3 . También suele aparecer de manera muy similar a un quiste odontogénico no neoplásico y con frecuencia se diagnostica erróneamente como un quiste dentígero o un queratoquiste odontogénico. Por lo tanto, la confirmación histológica mediante la realización de una biopsia es obligatoria en estos casos $^{5}$. Según los datos en la literatura, los patrones foliculares se presentan en un $24.8 \%$ de los casos y son plexiformes en el $24.7 \%$; ambos fueron los patrones histopatológicos más comunes. Los tumores acantomatoso (5.7\%), los patrones de células granulares (2.5\%) y de células basales (0.4\%) fueron raros según Faqui ${ }^{8}$.

Los ameloblastomas pueden presentarse en radiografías convencionales como una radiolucidez cortical unilobular o multilobular que se asemeja a un quiste. Los septos óseos pueden dar como resultado una apariencia de panal. La expansión bucal y lingual es más común en el ameloblastoma que en los tumores odontogénicos queratoquísticos. La reabsorción de las raíces puede o no estar presente. El diagnóstico diferencial radiográfico incluye: un tumor odontogénico queratoquístico, un mixoma odontogénico, así como tumores y quistes no odontogénicos, como un granuloma central de células gigantes y un quiste óseo simple, respectivamente $2,5,6,10$.

El tratamiento del ameloblastoma es controversial, en donde muchos autores prefieren la extirpación quirúrgica amplia para evitar recidivas ${ }^{6,9}$ y el manejo conservador con terapias coadyuvantes ${ }^{11,12}$. El objetivo del estudio es relatar el éxito de la técnica conservadora en estos tumores, con un buen resultado estético y funcional; en dependencia de la variante clínica, la extensión tumoral y la edad del paciente. Se describen las circunstancias relevantes del abordaje quirúrgico empleado en relación con el legrado y la ostectomía periférica, sin evidencia de recidiva desde el año 2011 al 2019.

Previo a la presentación del caso y seguimiento clínico-radiográfico de 8 años, se obtuvo el consentimiento informado del paciente, además se cuenta con el acta de aprobación del comité de ética del Hospital Nacional Rosales.

\section{Presentación del caso}

Se presenta un joven masculino de 13 años de edad que acude al servicio de cirugía maxilofacial del Hospital Nacional Rosales, porque desde hacía 6 meses empieza a notar un aumento de volumen de la región submandibular, que describió como progresivo e indoloro. A la historia clínica el paciente no tenía antecedentes médicos, quirúrgicos o alergias que fueran contributorias al caso. Al examen clínico extraoral, el paciente tenía un aumento de volumen visible de aproximadamente $4 \times 4 \mathrm{~cm}$ de diámetro en la región submandibular izquierda (Figura 1a.). En la región mandibular izquierda se palpa crepitación y dolor.

El examen intraoral reveló tumefacción en la región posterior del lado izquierdo de 


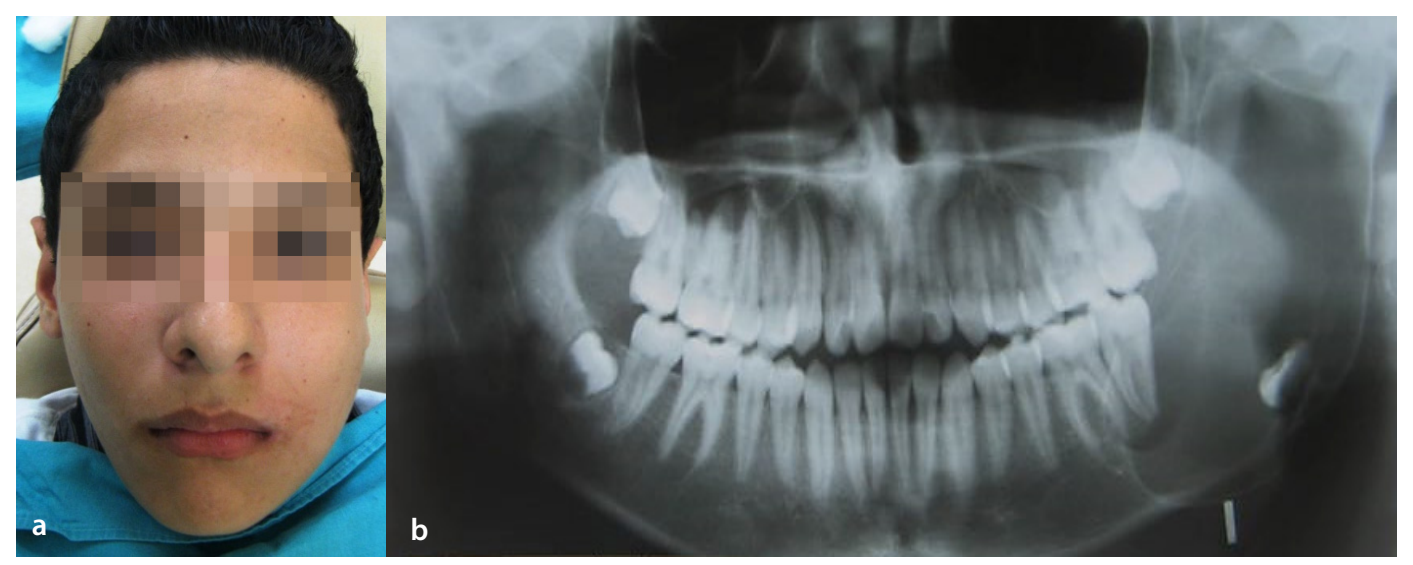

Figura 1. a. Paciente 13 años con aumento de volumen submandibular. b. Lesión radiolúcida unilocular en la región del ángulo mandibular izquierdo de $5 \times 3 \mathrm{~cm}$. de diámetro.

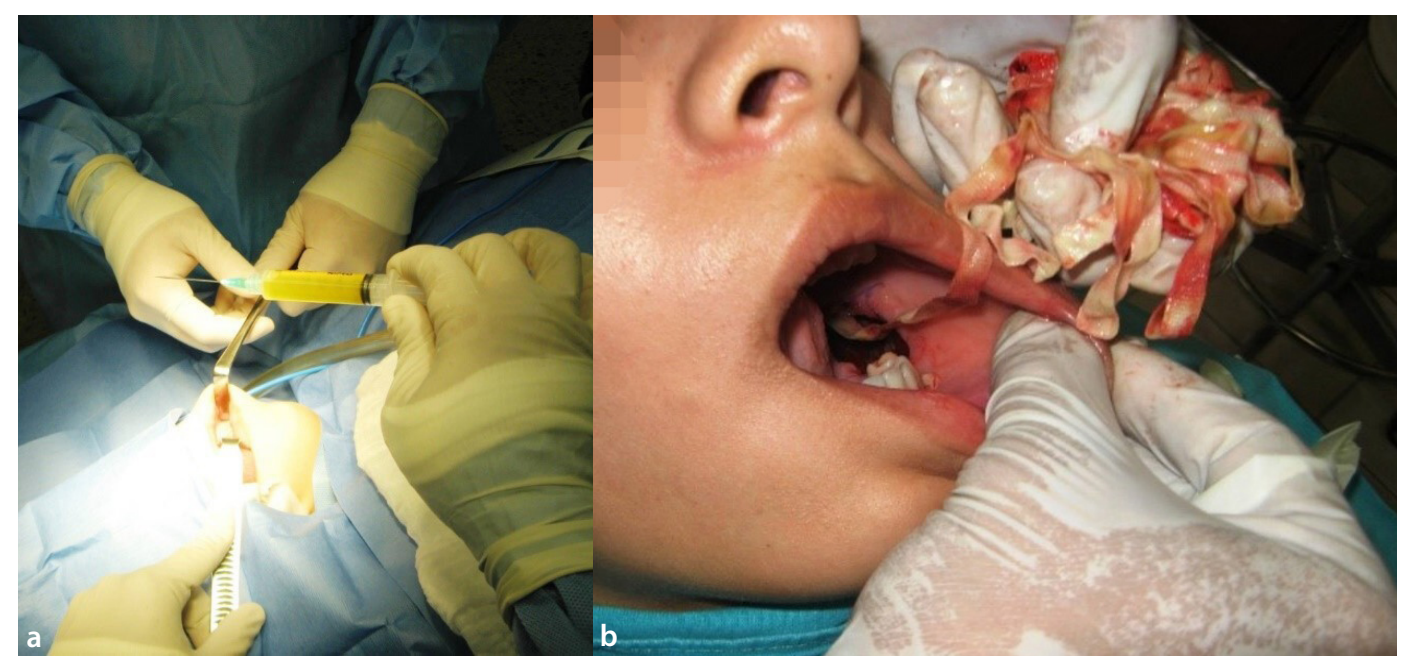

Figura 2. a. Citología aspirativa con aguja fina (CAAF) presentó contenido líquido de color amarillento. b. Cambio de mecha con nitrofurazona al 0.2\%;1 vez por semana.

la mandíbula, causando desplazamiento lingual y movilidad grado 2 , de la primera y segunda molar permanente inferior izquierda (36-37).

Al examen radiográfico se pudo evidenciar una extensa lesión radiolúcida unilocular en la región del ángulo mandibular izquierdo, que se extendía hasta la escotadura sigmoidea. No presentó reabsorción radicular de la primera y segunda molar permanente inferior izquierda \#36 y 37 . Pero sí presentó desplazamiento del germen del diente 38 hacia la base de la mandíbula, cerca del ángulo mandibular (Figura 1b).

El diagnóstico diferencial en este caso fue queratoquiste odontogénico, ameloblastoma de tipo uniquístico, quiste dentígero y lesión central de células gigantes. Al realizarle la citología aspirativa con aguja fina (CAAF), se obtuvo un líquido con aspecto amarillento, que reportaba presencia de histiocitos, leucocitos polimorfonucleares y material negativo a malignidad (Figura 2a).

Se realiza la toma de biopsia excisional que de acuerdo con las características al microscopio confirmaron que se trataba de un ameloblastoma de la región mandibular izquierda.

Se procedió al tratamiento quirúrgico, que consistió en enucleación del tumor y marsupialización, con curetaje, ostectomía periférica, y extracción de primera, segunda $y$ tercera molar permanentes inferiores izquierda \#36-38.

Posteriormente se realizó cambios de mechas impregnadas con nitrofurazona al $0.2 \%$, una vez por semana durante 2 meses (Figura 2b). Luego se colocó un obturador acrílico temporal para facilitar la cicatrización y la regeneración ósea.

Debido a que el ameloblastoma se podría comportar como un tumor agresivo, con una alta tasa de recurrencia, el paciente fue monitorizado mediante controles clínicos frecuentes para realizar radiografía de la evolución. A los 3 meses de operado, el paciente se presentó a la consulta con buena cicatrización de la herida operatoria intraoral y radiográficamente no presentaba cambios óseos (Figura 3) 


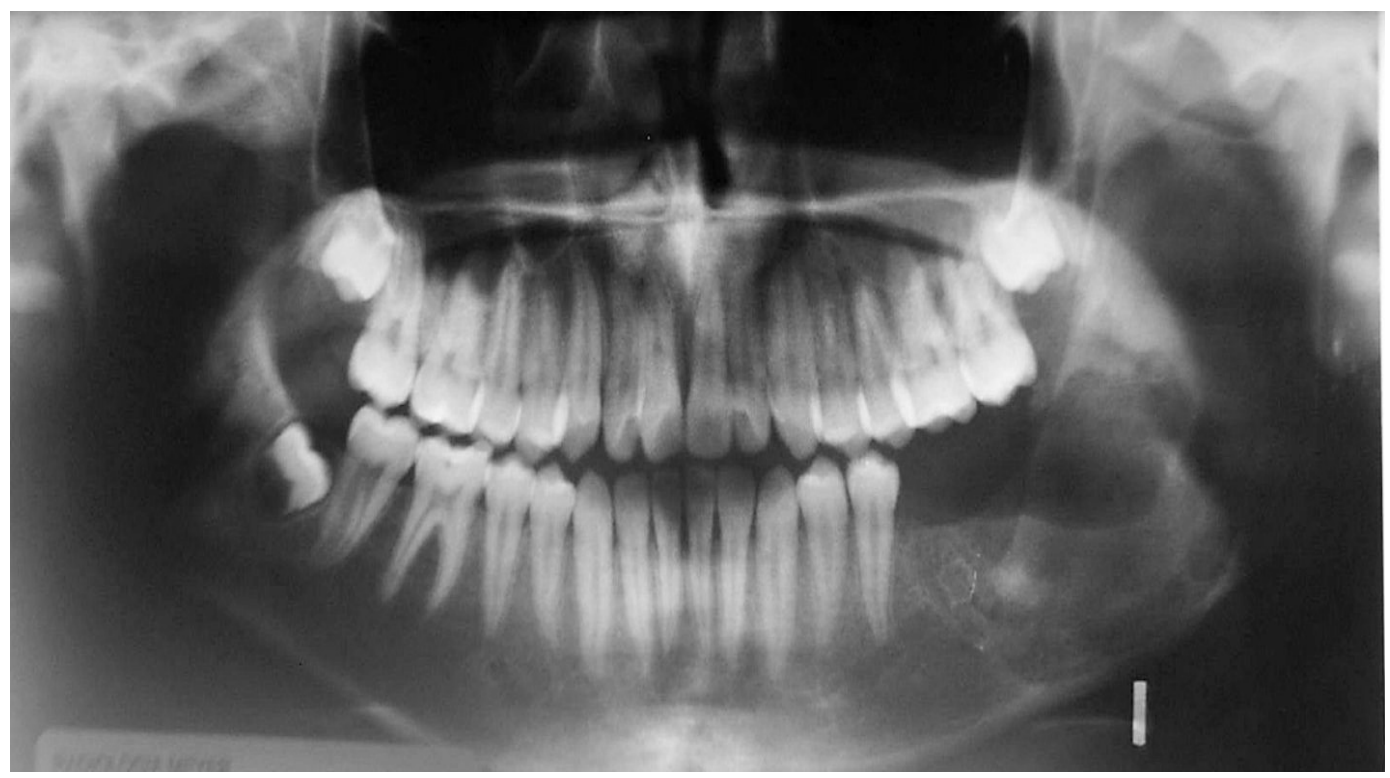

Figura 3. Radiografía panorámica a los 3 meses de la operación, evaluación de control.

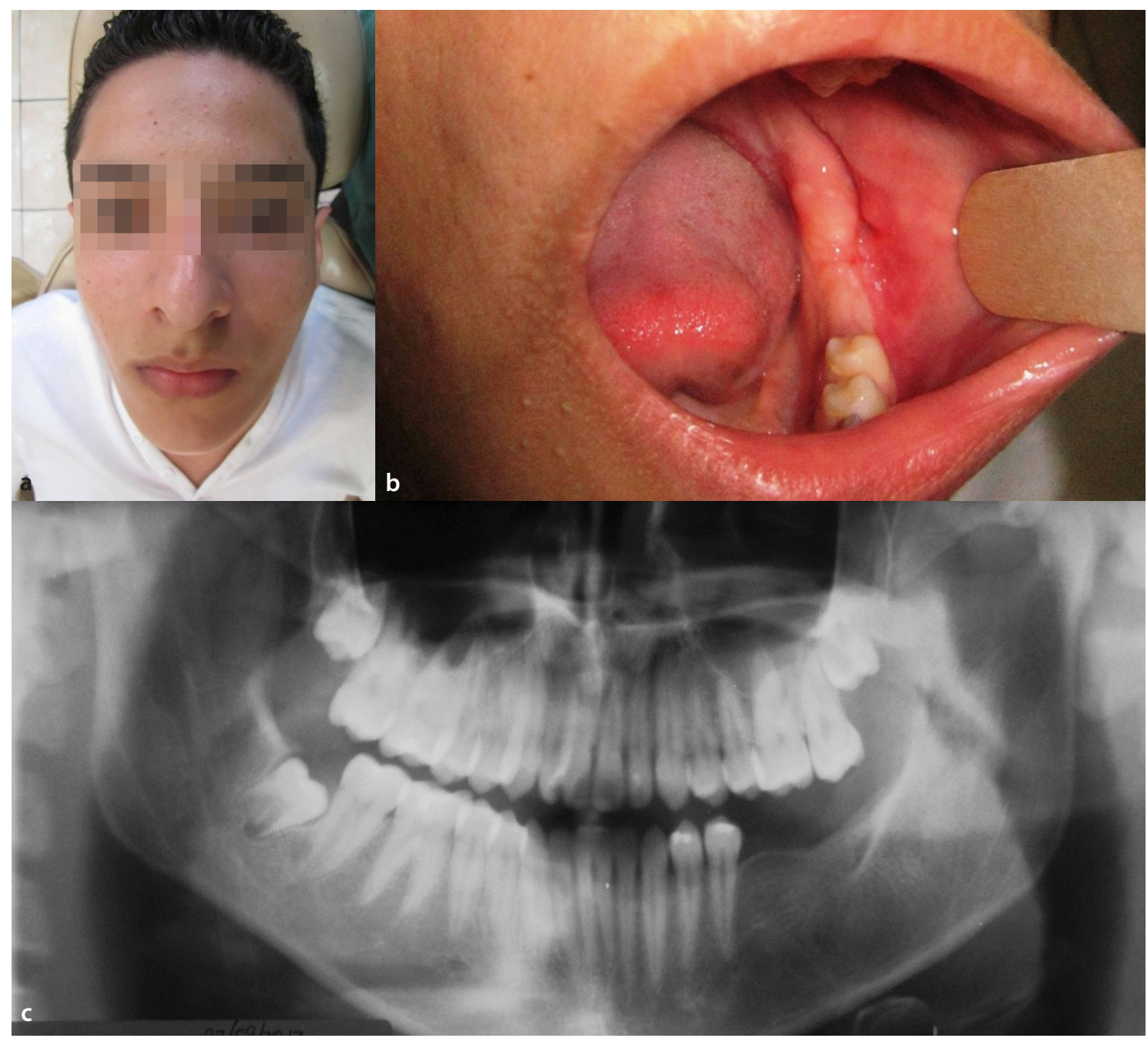

Figura 4. a. Paciente sin asimetrías clínicas. b. Cicatrización de tejido blando. c. Radiografía de control al año y medio de postquirúrgico.

En la cita de control, después de un año y 7 meses del postquirúrgico, se presentó el paciente con buen estado general, sin asimetría facial (Figura 4a). Al examen intraoral se podía observar la lesión cicatrizada (Figura 4b) y buena regeneración ósea mandibular (Figura 4c).
En la consulta de seguimiento a los 3 años, se decide que el paciente inicie tratamiento ortodóntico por la extrusión de la primera y segunda molar permanente superior izquierda, sumado a una retroinclinación de los incisivos inferiores y una mordida cruzada anterior. Para el manejo del cuadro se 


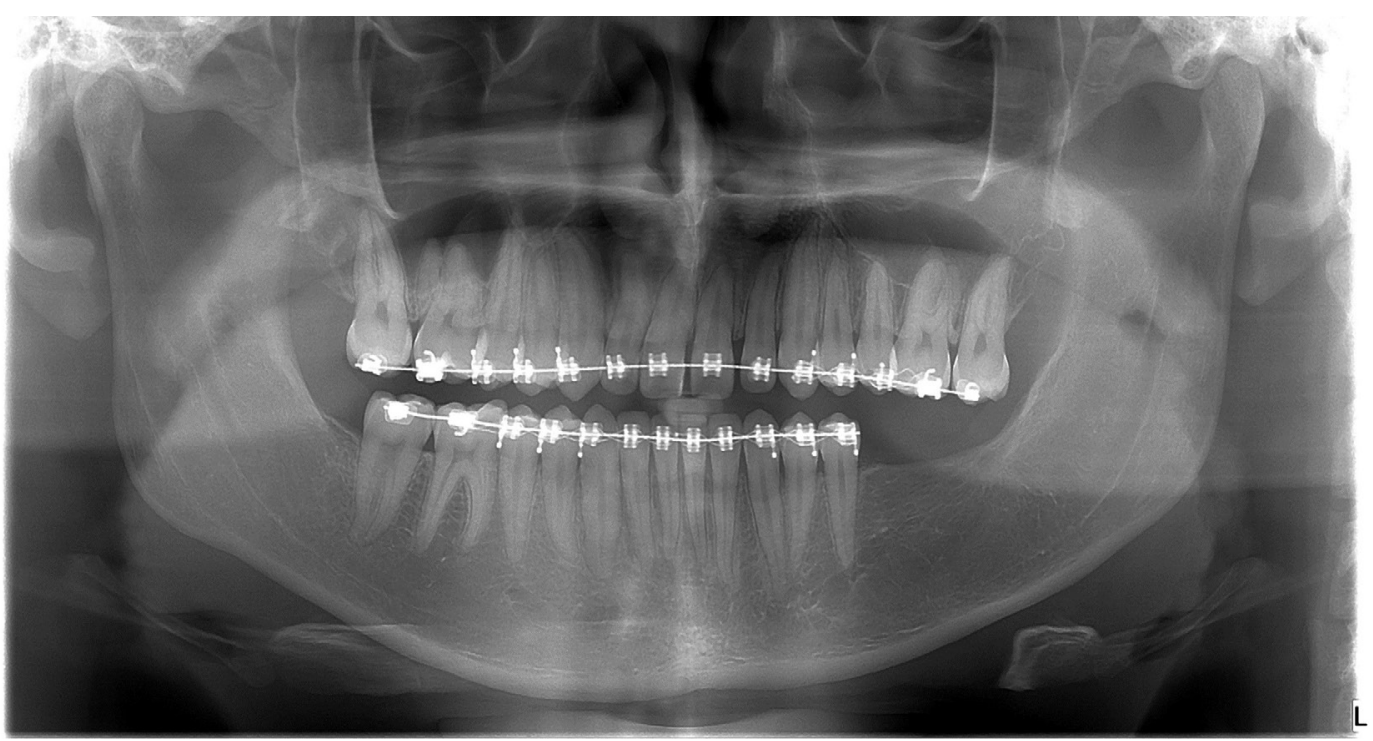

Figura 5. Paciente con tratamiento ortodóntico, 3 años del postquirúrgico.
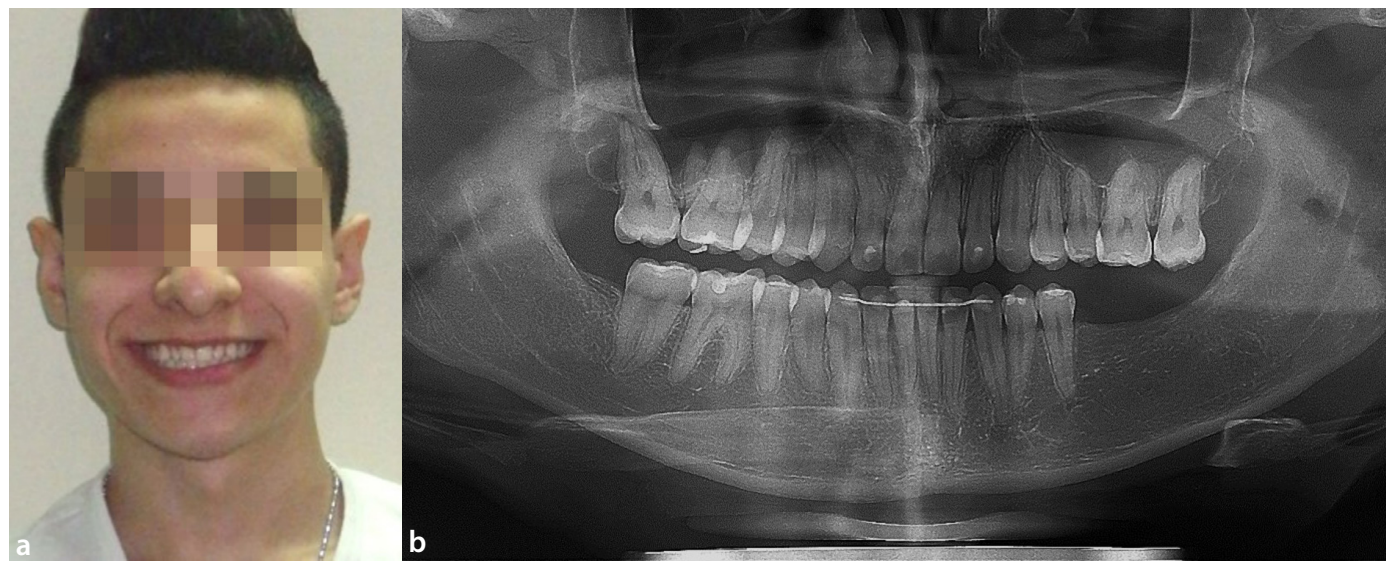

Figura 6. a. Foto Frontal, sin evidencia de asimetrías faciales. b. Regeneración ósea fisiológica normal, finalizado tratamiento ortodóntico.

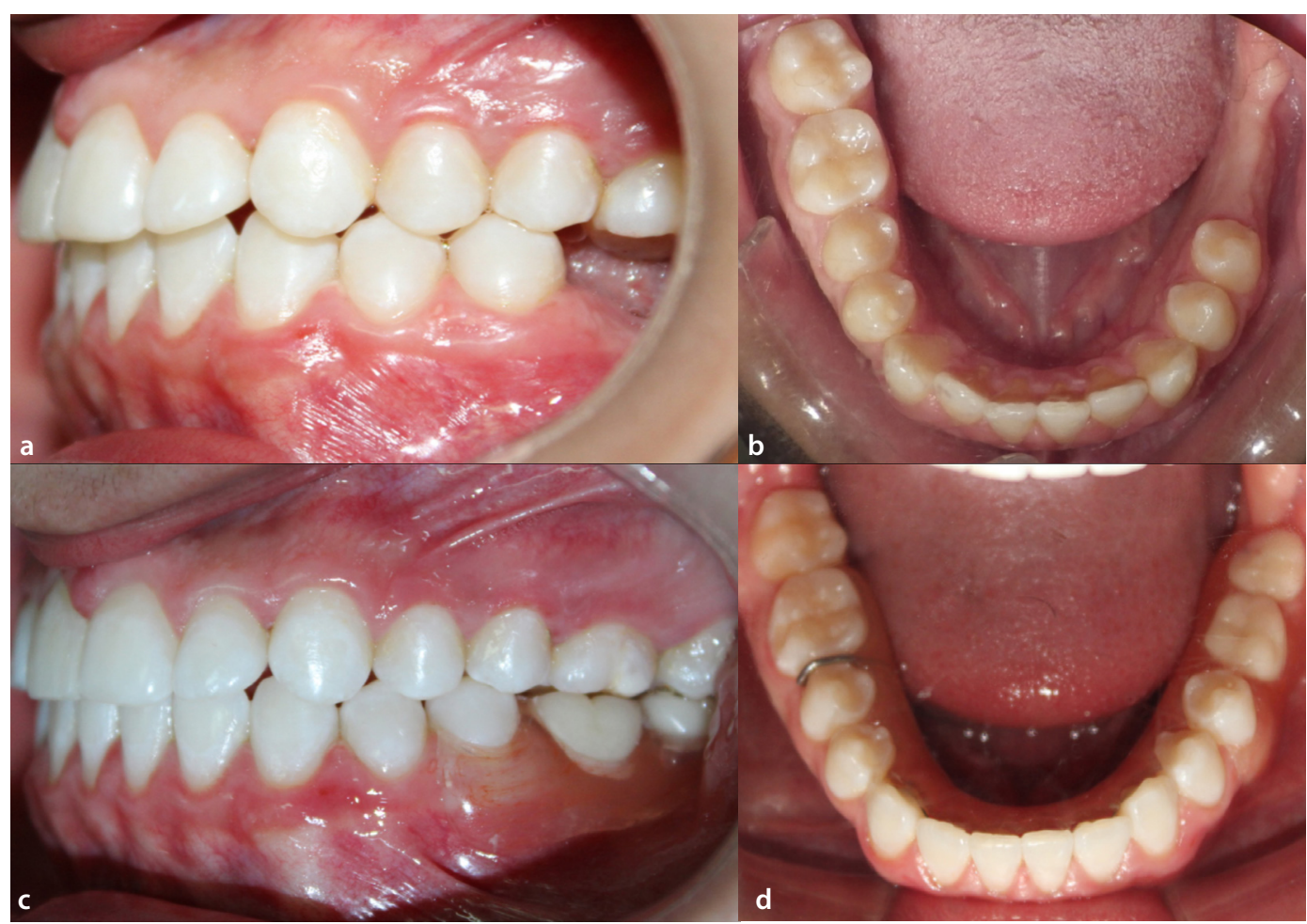

Figura 7. a. y b. Evaluación clínica intraoral, donde no se evidencia lesión patológica. c y d. Fotos intraorales con buenas relaciones oclusales y prótesis removible. 


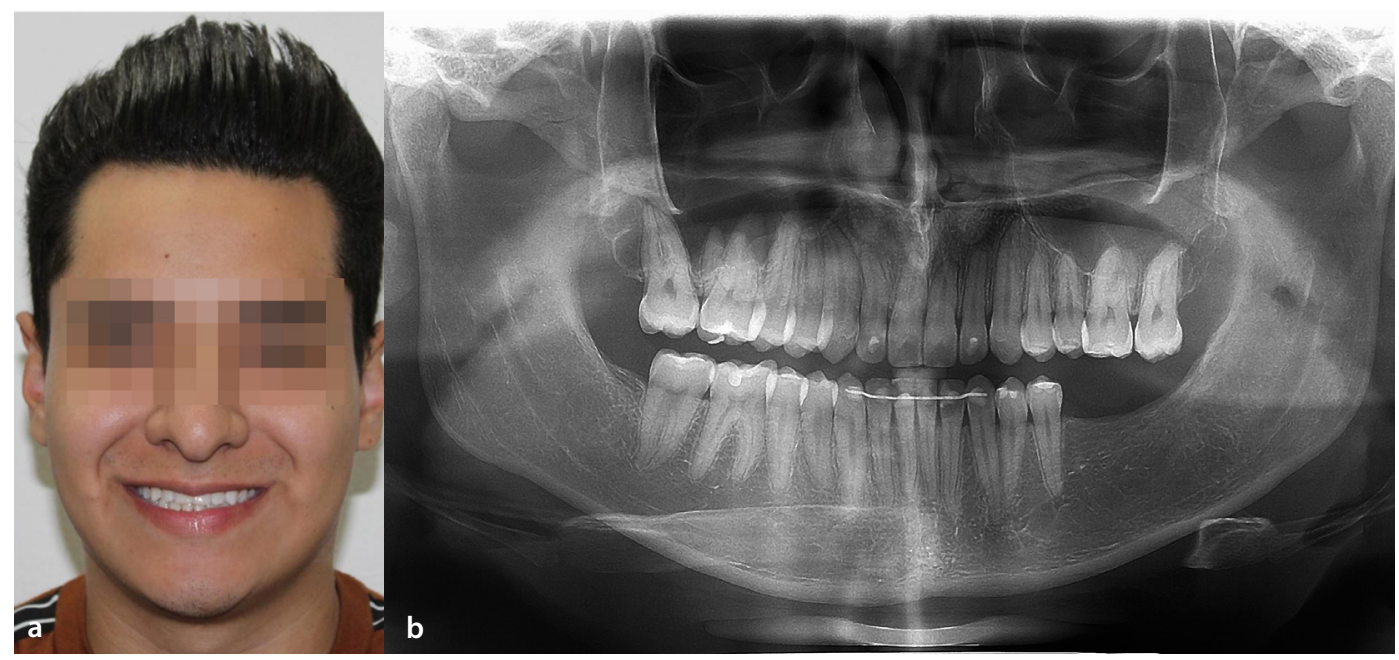

Figura 8. a. Paciente asintomático. b. Radiografía panorámica control 8 años.

realiza extracción de terceros molares retenidas (cordales) superiores e inferior (Figura 5).

Se observa paciente a los 7 años de operado con examen clínico y radiográfico normal, posterior a tratamiento ortodóntico (Figura 6a-6b).

Actualmente el paciente cuenta con su prótesis parcial removible de tipo valplast (Figura 7d).

El paciente fue visto en su último control en marzo del año 2020. Con buen estado general, sin sintomatología de patología a nivel maxilar. Tenía un plan quirúrgico y se encontraba a la espera de colocación de implantes dentales (Figura 8a-8b).

\section{Discusión}

En general, los ameloblastomas se caracterizan por agresividad a nivel local. Los tratamientos conservadores, como la marsupialización, la enucleación y el legrado, al tiempo que preservan la integridad ósea y permiten el crecimiento continuo de la mandíbula, parecen estar asociados con una alta tasa de recurrencia. Algunos artículos informan altas tasas de recurrencia para ameloblastomas que no fueron tratados radicalmente ${ }^{6,9}$.

El enfoque terapéutico del ameloblastoma debe analizarse después de un análisis exhaustivo de diferentes factores ${ }^{3}$ : tamaño y localización, características clínicas, tasa de crecimiento, relación con estructuras vecinas, histología, presentación clínica de la recurrencia, edad del paciente y condiciones generales.

A pesar de que en la literatura el tratamiento de resección con un margen de 1-1.5 cm de hueso aparentemente de tejido sano, evita recidivas ${ }^{4,6}$. Muchos consideran que la resección quirúrgica debería considerarse cuando la afección se repite más de dos veces o cuando el paciente lo solicite ${ }^{13}$ y no ser adoptado un enfoque quirúrgico más agresivo de entrada; o únicamente por tener el material y los recursos para reconstrucción inmediata de las mandíbulas, así como la disposición de rehabilitación protésica mediante el uso de implantes dentales ${ }^{6}$. Debido a este análisis y para favorecer al joven, el servicio de cirugía maxilofacial del Hospital Rosales tomó la decisión de optar por el tratamiento conservador, valorando su variante clínica uniquístico, sus características radiográficas, edad del paciente, en lugar de un tratamiento invasivo.

Cuando el afecto es un paciente joven, el criterio de tratamiento se torna distinto, debido a que deben considerarse factores fisiológicos de la edad, por ejemplo ${ }^{13}$ : el crecimiento facial continuo y la fisiología ósea diferente (hueso más esponjoso, aumento del recambio óseo y periostio reactivo), la presencia de piezas dentales no erupcionados y por la dificultad en el diagnóstico inicial.

Otro factor importante en cuanto a la selección de un tratamiento radical e invasivo, es que este puede presentar traumas psicológicos serios, debido a la etapa de desarrollo temprana en que se presenta la enfermedad ${ }^{4,12-14}$.

Cuando se ha retirado quirúrgicamente un quiste, la cicatrización ósea es una etapa muy importante para la rehabilitación estética y funcional de los maxilares. El tratamiento conservador cumple con requisitos para la regeneración ósea espontánea. Estas son ${ }^{15}$ : mantenimiento de la cavidad o defecto, para permitir la invasión vascular, celular y proliferativa; y para promover el reposo funcional gracias a la ausencia de carga mecánica.

Analizando la regeneración ósea, luego de la enucleación de este ameloblastoma, se controló en los primeros meses; así como años posteriores a la cirugía, que se presentaron aumentos significativos de la 
densidad del hueso, sin necesidad de materiales de injerto, lo que puede dar la opción al paciente de poderse rehabilitar protésicamente.

Cabe aclarar la elección de los tratamientos para ameloblastoma no siempre permiten elegir un método conservador; será dependiente de las variantes clínicas, subtipo histológico, localización, la edad del paciente, entre otros aspectos. Ambas cirugías conservadora o agresiva se determinarán según individualización de cada caso.

\section{Conclusiones}

En este reporte de caso de un paciente joven en el servicio de cirugía maxilofacial se tomó la decisión de manejo conservador por su variante clínica, edad del paciente y por la localización del ameloblastoma; con el objetivo de no someter al riesgo de una intervención agresiva, con el consiguiente impacto psicológico. El éxito de este caso clínico ha sido la ausencia de recaída en 8 años de seguimiento, gracias a la colaboración del paciente y su familia. De no contar con ello, la decisión podría inclinarse a ser radical, pues las recidivas suelen ser más agresivas. En estos casos es vital el seguimiento después del tratamiento quirúrgico, por las posibles recurrencias, incluso después de 5 años tratamiento.

\section{Referencias bibliográficas}

1. Silva JN da, Santos CN dos, Rocha AC, Carli ML de, Hanemann JAC, Pereira AAC. Extensive ameloblastoma in young patient: 5-year follow-up with no recurrence using conservative treatment. RGO - Rev. Gaúcha Odontol. 2018;66(2):181-186. DOI: 10.1590/19818637201800020000133357

2. Adel K. El-Naggar JKCC. WHO Classification of Head and Neck Tumours. 4th Edition. 2017.

3. Pozo JA, Espinoza J. Ameloblastoma uniquístico, bases del tratamiento conservador. Presentación de caso clínico y actualización bibliográfica. Rev. Esp. Cir. Oral Maxilofac. 2010;32(2):88-91. DOI: 10.1016/ S1130-0558(10)70022-8

4. Isolan C, Moreira A, Edges A, Post L. Successful conservative treatment of a mandibular unicystic ameloblastoma: 13-year follow-up. J. Clin. Exp. Dent. 2018;10(11):1123-1126. DOI: https://doi. org/10.4317/jced.54897
5. Kim J, Nam E, Yoon S. Conservative management (marsupialization) of unicystic ameloblastoma: literature review and a case report. Maxillofac Plast Reconstr Surg. 2017. DOI: 10.1186/s40902-017-0134-0

6. 6. Hertog D, Waal I Van Der. Ameloblastoma of the jaws: A critical reappraisal based on a 40-years single institution experience. Oral Oncol. 2010;46(1):61-64. DOI: 10.1016/j. oraloncology.2009.11.002

7. Sakuranaka H, Sekine A, Miyamoto I, Yamakawa Y, Hirata A, Hagiwara E, et al. Pulmonary Malignant Ameloblastoma without Local Recurrence 31 Years after Primary Resection: A Case Report and Literature Review. Intern. Med. 2020:1-4. DOI: 10.2169/internalmedicine. 3716-19

8. Hendra FN, Cann EM Van, Helder MN, Ruslin $M$, Visscher JG De,et al. Global incidence and profile of ameloblastoma : A systematic review and meta-analysis. Wiley Online Libr. 2019;(October 2018):1-10. DOI: $10.1111 /$ odi.13031

9. Hendra FN, Sabrina D, Kalla N, Cann EM Van, Vet HCW De,et al. Radical vs conservative treatment of intraosseous ameloblastoma: systematic review and meta-analysis. Wiley Online Libr. 2018;31(0):0-2. DOI: $\underline{10.1111 /}$ odi.13014

10. Kreppel M, Zöller J. AmeloblastomaClinical, radiological, and therapeutic findings. Oral Dis. 2018;24(1-2):63-66. DOI: $10.1111 /$ odi.12702

11. Siriwardena BSMS. Unicystic ameloblastoma: Analysis of 370 cases in a single center, Sri Lanka. J. Oral Pathol. Med. 2018:0-2. DOI: $\underline{10.1111 / \text { jop. } 12740}$

12. Takahashi K, Miyauchi K, Sato K, Case CR. Treatment of ameloblastoma in children. Br. J. Oral Maxillofacud Surg. 1998:453-456.

13. Seintou A. Unicystic ameloblastoma in children: systematic review of clinicopathological features and treatment outcomes. Int. J. Oral Maxillofac. Surg. 2014;(3):1-8. DOl: 10.1016/j.ijom.2014.01.003

14. BaldasseriniG, Scomparin LF. Epidemiological profile of ameloblastoma affected patients subjected to surgery at a tertiary hospital in the state of Sao Paulo. Rev. Odontol. Mexicana. 2018;22 (2):82-87.

15. Ricardo JH. Cicatrización y regeneración ósea de los maxilares después de una quistectomía: reporte de un caso y revisión de la literatura Healing and Bone Regeneration of the Jaws Cystectomy Post:Case Report and Literature Review. Rev. Odontol. 2011;30(65):71-78. 